\title{
INFLUÊNCIA DO CLIMA REGIONAL SOBRE O CLIMA LOCAL A PARTIR DO DIAGNÓSTICO DE ABRANGÊNCIA ESPACIAL E EXTRAPOLAÇÃO ESCALAR
}

\author{
GOBO, João Paulo Assis- jpgobo@usp.br \\ Universidade de São Paulo / USP \\ GALVANI, Emerson - egalvani@usp.br \\ Universidade de São Paulo / USP
}

\author{
WOLLMANN, Cássio Arthur - cassio_geo@yahoo.com.br \\ Universidade Federal de Santa Maria / UFSM
}

\begin{abstract}
RESUMO: O objetivo geral desta pesquisa foi determinar a abrangência espacial do Índice Subtropical Brasileiro (ISB), desenvolvido por Gobo (2018) em Santa Maria-RS, para outras localidades do estado do Rio Grande do Sul, transpondo dados climáticos obtidos na escala local para a escala regional. Analisou-se estatisticamente a influência do clima regional sobre o clima local por meio de uma análise de agrupamento (cluster) para dados de temperatura do ar, umidade relativa do ar e velocidade do vento de nove estações meteorológicas, durante os mesmos dias e horários dos trabalhos de campo realizados em Santa Maria. Dessa forma, iniciou-se o processo de análise de agrupamento dos dados das estações meteorológicas que apresentassem similaridade, sendo definidas as variáveis a serem agrupadas. Foram geradas matrizes de proximidade no programa SPSS statistic 21 e posteriormente introduzidas no programa Multidendograms 2.1.0 para a elaboração dos dendrogramas de agrupamento. Após validações por meio cálculo do coeficiente cofenético, foram geradas duas análises de componentes principais, uma contendo a temperatura do ar, a velocidade do vento e a umidade relativa do ar como variáveis e a outra contendo estas três variáveis mais o Índice Subtropical Brasileiro (ISB). Os resultados mostraram que, devido aos baixos coeficientes de correlação cofenética, apenas para o agrupamento da variável temperatura do ar houve um número maior de estações meteorológicas agrupadas, sendo um grupo com duas estações e outro com sete estações meteorológicas homogêneas. Também se verificou um grupo com duas estações meteorológicas na análise de agrupamento para a umidade relativa do ar; um grupo contendo três estações meteorológicas para o agrupamento da variável ISB; e um grupo com quatro estações meteorológicas na análise de agrupamento para a velocidade do vento. Por outro lado, a umidade relativa agrupa-se com praticamente todas as estações meteorológicas analisadas. Por fim, concluiu-se que os resultados encontrados evidenciam a influência da escala temporal de análise na transferência de informações de escalas superiores para escalas inferiores do clima.
\end{abstract}

PALAVRAS-CHAVES: Abrangência espacial, Índice Subtropical Brasileiro, extrapolação escalar, clima regional e local, Rio Grande do Sul.

\section{INFLUENCE OF THE REGIONAL CLIMATE ON LOCAL CLIMATE FROM THE SPACE SCOPE AND SCALAR EXTRAPOLATION DIAGNOSIS}

ABSTRACT: This research concerns in to determine the spatial comprehensiveness of the Brazilian Subtropical Index (BSI), developed by Gobo (2018) in Santa Maria-RS, in order to expand it to other locations in the state of Rio Grande do Sul, trying to do a local to regional scale data expansion. The influence of the regional climate on the local climate was statistically analyzed by means of a cluster analysis for data on air temperature; relative air humidity and wind speed of nine weather stations in Rio Grande do Sul, during the same days and hours of the fieldwork in Santa Maria. Then, the process of clustering the data of weather stations that presented similarity was started, and the variables to be grouped were defined. Proximity matrices were generated in SPSS statistic 21 program and introduced in the Multidendograms 2.1.0 program for the 
elaboration of clustering dendrograms. After validations, cophenetic correlation coefficient was calculated, and two principal component analyzes were generated, one containing the air temperature, the wind speed and the relative humidity of the air as variables and the other containing these three ones. Results proved that, due to the low coefficients of coefonet correlation, only for the grouping of the air temperature variable was a larger number of weather stations grouped, being a group with two stations, and one with seven homogeneous as well. There was also a group with two stations in the cluster analysis for relative humidity; a group containing three ones for grouping the BSI variable; and a group with four weather stations in cluster analysis for wind speed. In compensation, the relative humidity was grouped with practically all the weather stations analyzed. Finally, it was concluded that the results found evidence the influence of the temporal scale of analysis on the transfer of information from higher scales to lower scales of the climate.

KEYWORDS: Spatial comprehensiveness, Brazilian Subtropical Index, scalar extrapolation, regional and local climate, Rio Grande do Sul.

\section{INTRODUÇÃO}

Os estudos do clima atraem cada vez mais a atenção da comunidade científica devido à sua complexidade e importância, bem como às implicações socioeconômicas e à necessidade de prevenção e adaptação aos seus extremos. Porém, a complexidade de muitos desses estudos se deve às múltiplas escalas de espaço e de tempo que caracterizam os processos ativos no sistema climático, além dos fenômenos que eles originam, desde chuvas localizadas até grandes furacões (GHIL; CHEKROUN; STEPAN, 2015).

O clima global, em geral, e a temperatura do ar, em particular, variam em muitas escalas espaciais e temporais. Paluš (2014) sugere que nos dados de temperatura do ar de superfície analisados há uma manifestação regional de um fenômeno geral de interações em escala transversal, na dinâmica atmosférica, em que os modos globais de baixa frequência influenciam a variabilidade local e de alta frequência (JAJCAY et al., 2016). Como exemplo disso, Chekroun et al. (2011) afirmam que a fase dos modos de baixa frequência do El Niño Oscilação Sul (ENOS) influencia a variabilidade de alta frequência (ruído climático) da temperatura da superfície do mar no Pacífico tropical.

Em uma escala espacial muito menor, Krüger e Gonzalez (2016) analisaram a influência das alterações do albedo dos revestimentos de superfície sobre a temperatura do ar de cânions urbanos, em cidades de médio porte, localizadas nas oito zonas bioclimáticas brasileiras. Os autores demonstraram que o efeito do aumento local do albedo sobre a temperatura do ar não chega a ser efetivo, sendo a redução de temperatura do ar, induzida pelo uso de alto albedo, insuficiente para promover uma melhora no conforto térmico no plano do pedestre, o que de fato está associado diretamente com a influência do clima local sobre o microclima.

Jajcay et al. (2016) demonstraram a importância da compreensão da variabilidade climática nos modos regionais específicos da escala, suas interações de escala cruzada e relações causais com os modos de variação da circulação global que estão localizados não apenas no espaço (VEJMELKA et al., 2014), mas também em uma escala de tempo ou uma faixa de frequência (GROTH; GHIL, 2011; 2015).

Logo, fica evidente a importância da escala temporal na definição da escala espacial de análise e vice-versa, sendo a atmosfera caracterizada por 
fenômenos cujas escalas temporal e espacial têm grande amplitude de variações. Da mesma forma, as escalas espaciais são determinadas pelas dimensões características dos fenômenos, assim como as escalas temporais, pelo seu período de tempo (GRIMMOND; OKE, 1991).

Tratando-se da escala climática urbana, esta é muito mais abrangente quando se leva em consideração a escala temporal de análise (ORLANSKY, 1975; WANNER; FILLIGER, 1989; ANDRADE, 2003), como pode ser observado na Figura 1, que ilustra, ao contrário de diversas metodologias, a real ausência de limites rígidos para essas dimensões escalares

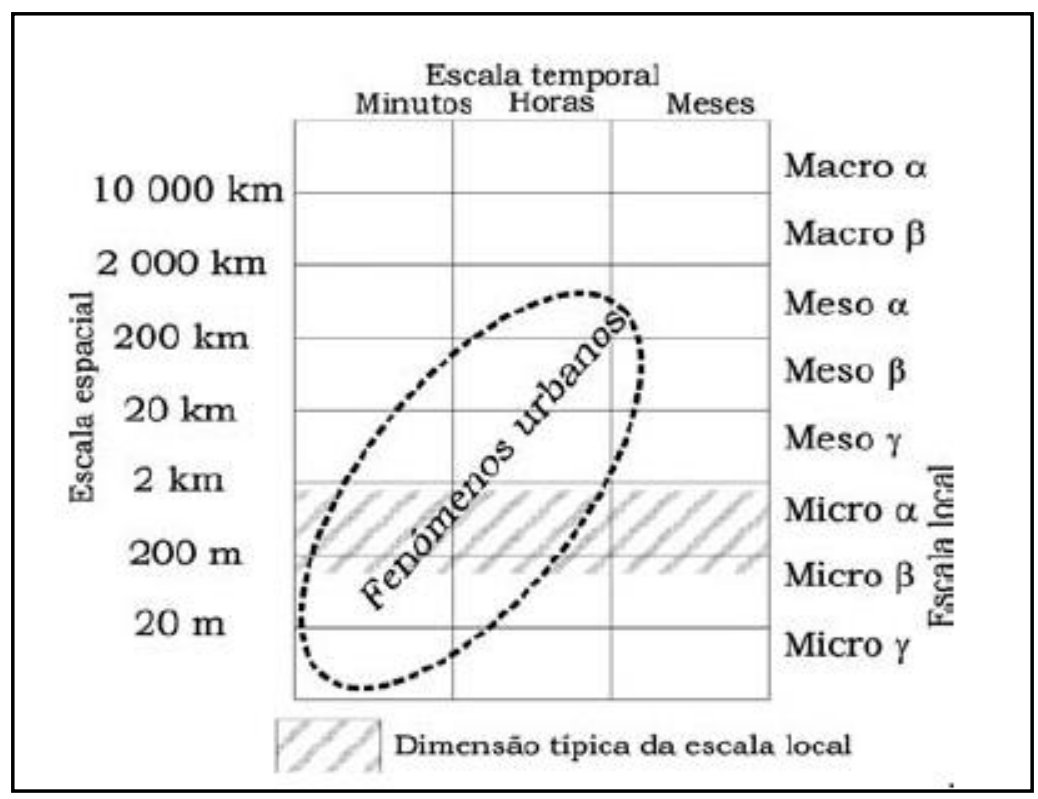

Figura 1 - Dimensão espaço/temporal da escala climática urbana. Fonte: Andrade (2005)

Segundo Andrade (2005), o tipo de tempo condiciona a variação espacial dos elementos climáticos no espaço urbano, sobretudo através do vento, da nebulosidade e da estabilidade vertical da atmosfera (LANDSBERG, 1981; OKE, 1987; ELIASSON, SVENSON, 2002; SVENSON, ELIASSON, 2002).

Logo, se sob fluxo sinótico intenso as diferenças locais são, em grande parte, suprimidas, em situação de estabilidade atmosférica os efeitos locais e microclimáticos controlam as condições climáticas da camada inferior da atmosfera (LANDSBERG, 1981).

Retrocedendo ao raciocínio levantado na revisão da literatura, cabe ressaltar o estudo de Ribeiro (1993) ao afirmar que o estabelecimento de limites rígidos para o microclima não traz resultados palpáveis, uma vez que o seu tamanho vai mesmo depender da natureza da superfície que lhe dá origem. Portanto, a transferência de informação de escalas maiores para menores, observada por Paluš (2014), torna-se uma base de sustentação para a hipótese levantada nesta pesquisa, mesmo o autor afirmando que essa transferência não se dá de escalas menores para escalas maiores. 
No entanto, será pressuposto que, havendo uma transferência de informação de escalas superiores para escalas inferiores do clima, e estas, como observado nos estudos de Jajcay et al. (2016), Chekroun et al. (2011) e Groth e Ghil $(2011 ; 2015)$, tendo a identificação dessa influência em consequência do período de análise (escala temporal) (PALUŠ, 2014), pretendeu-se nesta pesquisa determinar a abrangência espacial do Índice Subtropical Brasileiro, desenvolvido por Gobo (2018) em Santa Maria-RS, para outras localidades do estado do Rio Grande do Sul, extrapolando dados climáticos obtidos na escala local para a escala regional.

\section{MATERIAIS E MÉTODOS}

Para desenvolver uma análise de extrapolação escalar para o Índice Subtropical Brasileiro (GOBO, 2018), partiu-se das premissas anteriormente destacadas por Ribeiro (1993), Paluš (2014) e Landsberg (1981). Assim, optouse por analisar estatisticamente a influência do clima regional sobre o clima local a partir de uma análise de abrangência espacial, que foi elaborada por meio de uma análise de agrupamento (cluster) para dados de diferentes estações meteorológicas dispostas em distintos municípios do Rio Grande do Sul, durante os mesmos dias e horários dos trabalhos de campo em Santa Maria que determinaram a elaboração do Índice Subtropical Brasileiro (GOBO, 2018).

Observa-se na figura 1, a correlação entre a temperatura do ar da Estação Meteorológica Automática (EMA) localizada no centro de Santa Maria com a EMA-INMET localizada na região periférica da cidade foi elevada para todos os períodos de análise (verão e inverno). O que corrobora a hipótese de que, apesar do desvio da temperatura existente entre os dados obtidos no centro da cidade e os dados observados pela estação oficial afastada da área urbana, a temperatura em ambos os casos apresenta conformidade de comportamento. Logo, optou-se por utilizar os dados da EMA-INMET de Santa Maria para a avaliação de abrangência espacial.

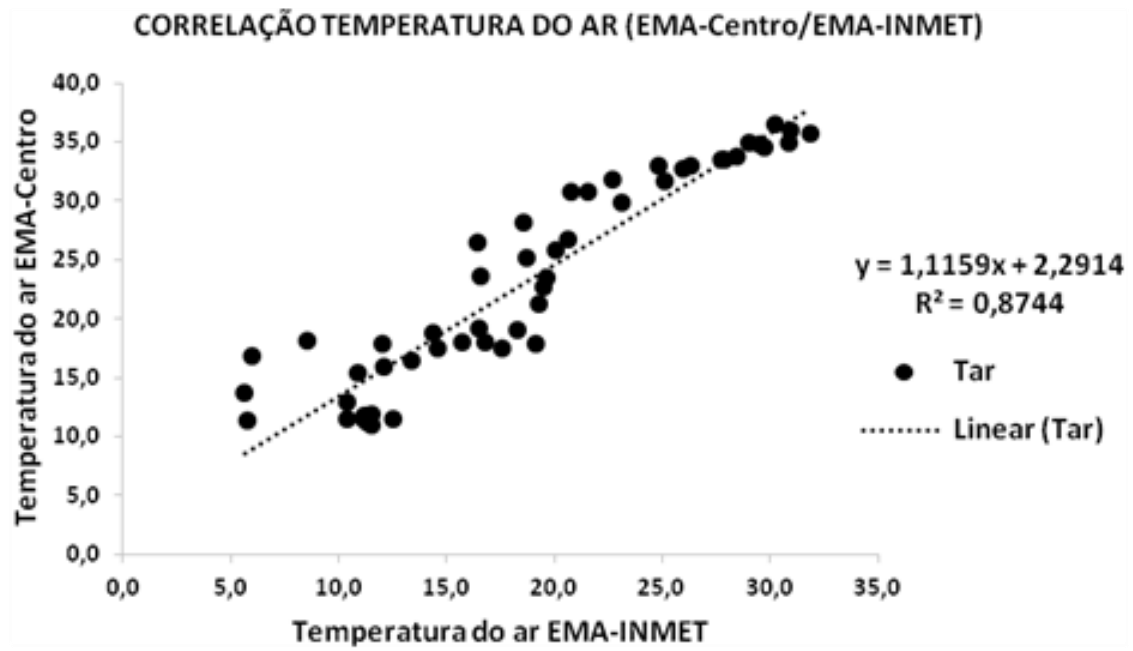

Figura 2 - Correlação entre os dados de temperatura do ar obtidos junto a EMA-INMET e da EMA-Centro. 
Assim, foram selecionados os dados de temperatura do ar, umidade relativa do ar e velocidade do vento de nove estações meteorológicas automáticas oficiais do INMET em diferentes pontos do estado do Rio Grande do Sul, respeitando o arranjo espacial destas em relação à distância de Santa Maria, as diferentes unidades geomorfológicas, segundo Müller Filho (1970), e as tipologias climáticas do estado, conforme classificação de Rossato (2014). São elas: Santa Maria; Caçapava do Sul; Rio Pardo; Jaguarão; Canela; Alegrete; Quaraí; Cruz Alta; Palmeira das Missões.

Não foram selecionadas estações meteorológicas localizadas em municípios da planície litorânea devido ao efeito da maritimidade, que condiciona a variabilidade da amplitude térmica diária e sazonal (MONTEIRO, 1999).

Os dados meteorológicos foram coletados no intervalo de uma hora, compreendendo o período das $8 \mathrm{~h}$ às $16 \mathrm{~h}$ dos dias 17,18 e 19 de janeiro de 2016 e o período das $9 \mathrm{~h}$ às $17 \mathrm{~h}$ dos dias 06, 07 e 08 de julho de 2016, respectivamente os mesmos dias e horários de coleta dos dados referentes aos períodos de verão e inverno da pesquisa de Gobo (2018), para a determinação do Índice Subtropical Brasileiro.

O ISB foi definido por Gobo (2018) a partir de uma regressão linear tendo como dados meteorológicos a temperatura do ar, a umidade relativa do ar e o vento, e uma escala de sensação térmica baseada no voto médio dos indivíduos entrevistados durante período de inverno e verão em Santa Maria (GOBO, 2018).

De posse dos dados, calculou-se o Índice Subtropical Brasileiro para cada uma das estações meteorológicas do INMET descritas, a partir da seguinte equação:

Equação 1

$$
I S B=0,1523 \times \operatorname{Tar}-0,0010 \times U R-0,4300 \times V-2,6425
$$

Em que: $\operatorname{Tar}=$ temperatura do ar $\left({ }^{\circ} \mathrm{C}\right) ; U R=$ umidade relativa do ar $(\%) ; V=$ velocidade do vento $(\mathrm{m} / \mathrm{s}) ; I S B=$ Índice Subtropical Brasileiro.

As faixas interpretativas do ISB são apresentadas a seguir na tabela 1 .

Tabela 1 - Faixas interpretativas para o Índice Subtropical Brasileiro (ISB).

\begin{tabular}{cl}
\hline$<=-3$ & Muito frio \\
$-2,9$ a -2 & Frio \\
$-1,9$ a -1 & Um pouco de frio \\
$-0,9$ a 0,9 & Nem frio nem calor \\
1 a 1,9 & Um pouco de calor \\
2 a 2,9 & Calor \\
$>=3$ & Muito calor \\
\hline
\end{tabular}

Fonte: Gobo (2018). 
Após o cálculo do ISB, iniciou-se o processo de análise de agrupamento, a qual, segundo Vicini (2005), constitui uma metodologia numérica multivariada, com o objetivo de propor uma estrutura classificatória ou de reconhecimento da existência de grupos, objetivando mais especificamente dividir o conjunto de observações em um número de grupos homogêneos, segundo algum critério de homogeneidade (REGAZZI, 2001).

O primeiro passo para realizar a análise de agrupamento foi formular o problema de aglomeração, no caso, agrupar estações meteorológicas que apresentassem similaridade nos dados de determinada variável. Em seguida foram definidas as variáveis em que iria se basear o agrupamento (Tar, UR, V e ISB) e, após esses processos, identificado o método mais adequado para a elaboração dos dendrogramas.

As matrizes de proximidade foram geradas no programa SPSS statistic 21 e posteriormente introduzidas no programa Multidendograms 2.1.0 para a elaboração dos dendrogramas de agrupamento. Os dendrogramas ou fenogramas representam uma síntese gráfica do trabalho desenvolvido, ou seja, resumem a informação e são de grande utilidade para a classificação, comparação e discussão dos agrupamentos (VICINI, 2005).

A forma mais adequada de validação do método utilizado na execução da análise de agrupamento é por meio do cálculo do coeficiente de correlação cofenético (VALENTIN, 2000; SARAÇLI; DOGAN; DOGAN, 2013), o qual determina o grau de deformação provocado pela construção do dendrograma. $O$ menor grau de distorção será refletido pelo maior coeficiente cofenético, fornecido pela matriz fenética $F$, na qual seus valores foram obtidos junto à matriz de distâncias inicial e pela matriz cofenética $C$, sendo estes os valores obtidos junto à matriz final das distâncias. O maior coeficiente cofenético possui a capacidade de evidenciar melhor a estrutura dos dados, isto é, a existência de grupos. No entanto, a maioria dos estudos utiliza o coeficiente cofenético 0,7 como limite de aceitação (VALENTIN, 2000; VICINI, 2005; SARAÇLI; DOGAN; DOGAN, 2013; MOREIRA et al., 2016). Assim, se o coeficiente de correlação cofenético for maior que $0,7,0$ método então empregado, bem como 0 agrupamento, são válidos.

Como o programa SPSS não calcula o coeficiente cofenético dos dendrogramas gerados, optou-se pela construção destes no programa Multidendograms 2.1.0, onde o cálculo do coeficiente de correlação cofenético é gerado automaticamente.

Após o teste dos principais métodos de agrupamento e do cálculo do coeficiente cofenético para cada um deles, determinou-se o método do encadeamento único (single linkage) como sendo o melhor método de agrupamento para as amostras e, consequentemente, o método que obteve os maiores valores de correlação cofenética. Assim, foram gerados agrupamentos para as médias horárias e diárias de cada variável. Segundo Vicini (2005), o método do encadeamento único baseia-se na distância mínima, regra do vizinho mais próximo, em que os dois primeiros objetos agrupados são os que apresentam menor distância entre si.

Além da escolha do método adequado, por meio do cálculo do coeficiente cofenético como medida de proximidade, a distância euclidiana foi usada para 
representar a semelhança e a dissimilaridade das estações analisadas. A distância euclidiana é comumente utilizada para a análise de variáveis quantitativas e corresponde à distância geométrica no espaço multidimensional entre observações (TERASSI; GALVANI, 2017). Segundo Regazzi (2001), embora a distância euclidiana seja uma medida de dissimilaridade, esta é, por vezes, referida como uma medida de semelhança, tendo em vista que quanto maior seu valor, menor a semelhança entre os indivíduos ou unidades amostrais.

A distância euclidiana é dada pela seguinte equação:

\section{Equação 2}

$$
D_{i i^{\prime}}=\left[\sum_{j=1}^{p}\left(X_{i j}-X_{i^{\prime} j}\right)^{2}\right]^{\frac{1}{2}}
$$

Por fim, após a análise de agrupamento e a elaboração dos dendrogramas para as variáveis observadas, optou-se pela execução de uma análise de componentes principais (PCA), por meio do programa The Unscrambler 9.7, a qual tem por objetivo descrever os dados contidos num quadro indivíduos/variáveis numéricas em que $p$ variáveis são mediadas com $n$ indivíduos (SARAÇLI; DOGAN; DOGAN, 2013). A análise PCA, assim como a análise de agrupamento, é uma análise multivariada e possibilita a identificação das medidas responsáveis pelas maiores variações entre os resultados.

Assim, foram utilizadas como conjunto de amostras as cidades estudadas e como variáveis a temperatura do ar, a velocidade do vento, a umidade relativa do ar e o índice ISB. A variável umidade relativa do ar foi normalizada na análise, já que seus valores numéricos eram muito discrepantes dos demais. Logo, tal variável foi corrigida pelo desvio padrão, para que as variáveis tivessem o mesmo peso na análise de componentes principais.

Por fim, foram geradas duas análises de componentes principais, uma contendo a temperatura do ar, a velocidade do vento e a umidade relativa do ar como variáveis e a outra contendo estas três variáveis já citadas e o Índice Subtropical Brasileiro.

\section{RESULTADOS E DISCUSSÃO}

\section{Análise de agrupamento e abrangência espacial}

A análise de agrupamento para as médias horárias das variáveis temperatura do ar, umidade relativa do ar, velocidade do vento e para o Índice Subtropical Brasileiro apresentou coeficientes de correlação cofenética baixos, porém os agrupamentos com o método do encadeamento único (single linkage) foram os que mostraram os melhores coeficientes, apesar de apenas o coeficiente cofenético para o agrupamento da variável velocidade do vento ter apresentado valor superior a 0,7 (Tabela 2). 
Tabela 2 - Coeficientes de correlação cofenética dos agrupamentos por método de encadeamento único para a média horária das variáveis observadas.

\begin{tabular}{cc}
\hline Agrupamentos (análise horária) & $\begin{array}{c}\text { Coeficiente de Correlação } \\
\text { Cofenético }\end{array}$ \\
\hline ISB & 0,130 \\
Tar & 0,115 \\
UR & 0,511 \\
V & $\mathbf{0 , 7 3 4}$ \\
\hline
\end{tabular}

Elaborada pelo autor.

Devido aos baixos coeficientes de correlação cofenética para os dados horários médios, os dendrogramas resultantes desses agrupamentos não apresentaram validação estatística, tampouco agrupamentos consistentes para os dados observados.

É possível notar nos dendrogramas da Figura 3 que a maioria das estações meteorológicas não formou agrupamento, o que caracteriza diferenças significativas entre elas.
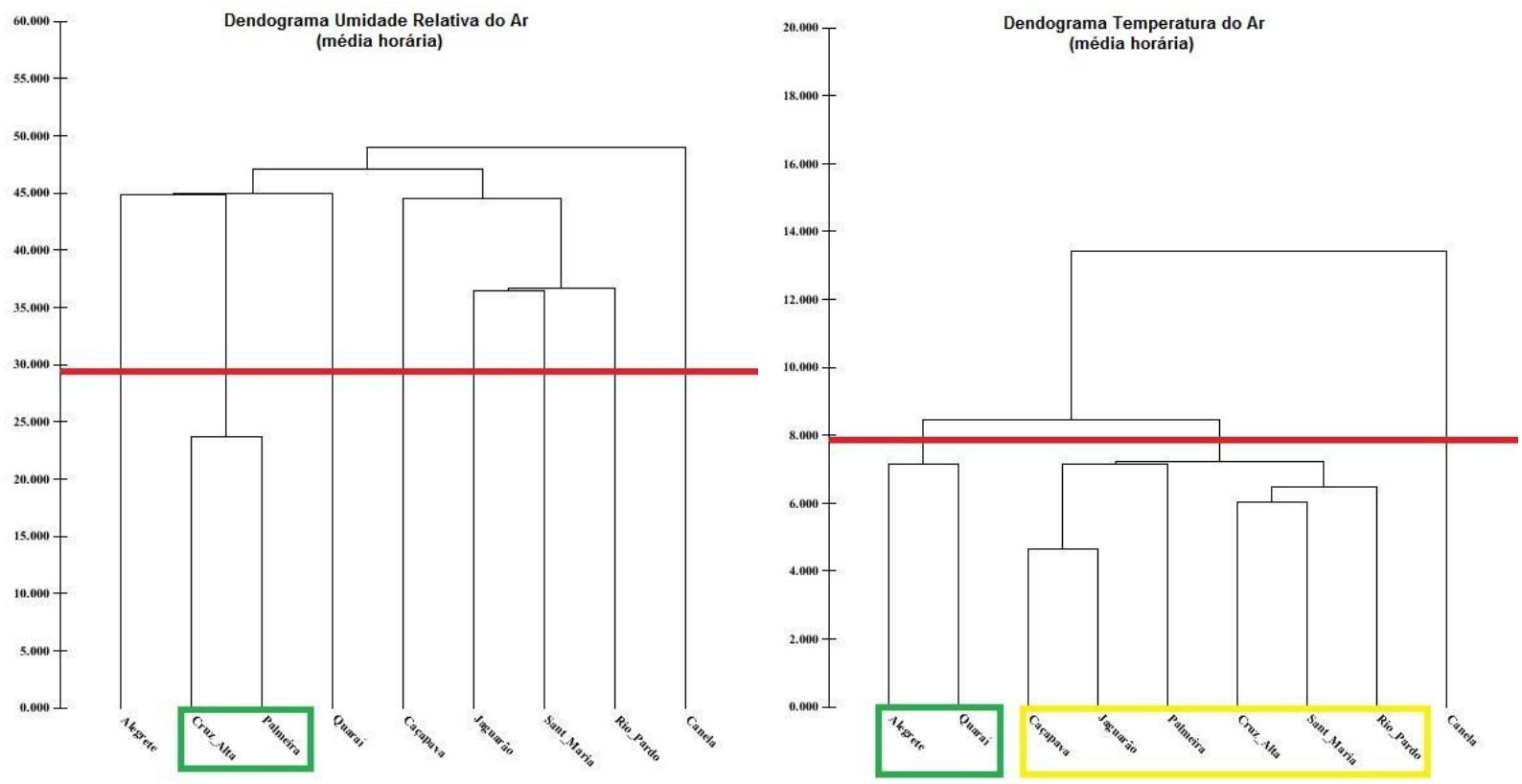

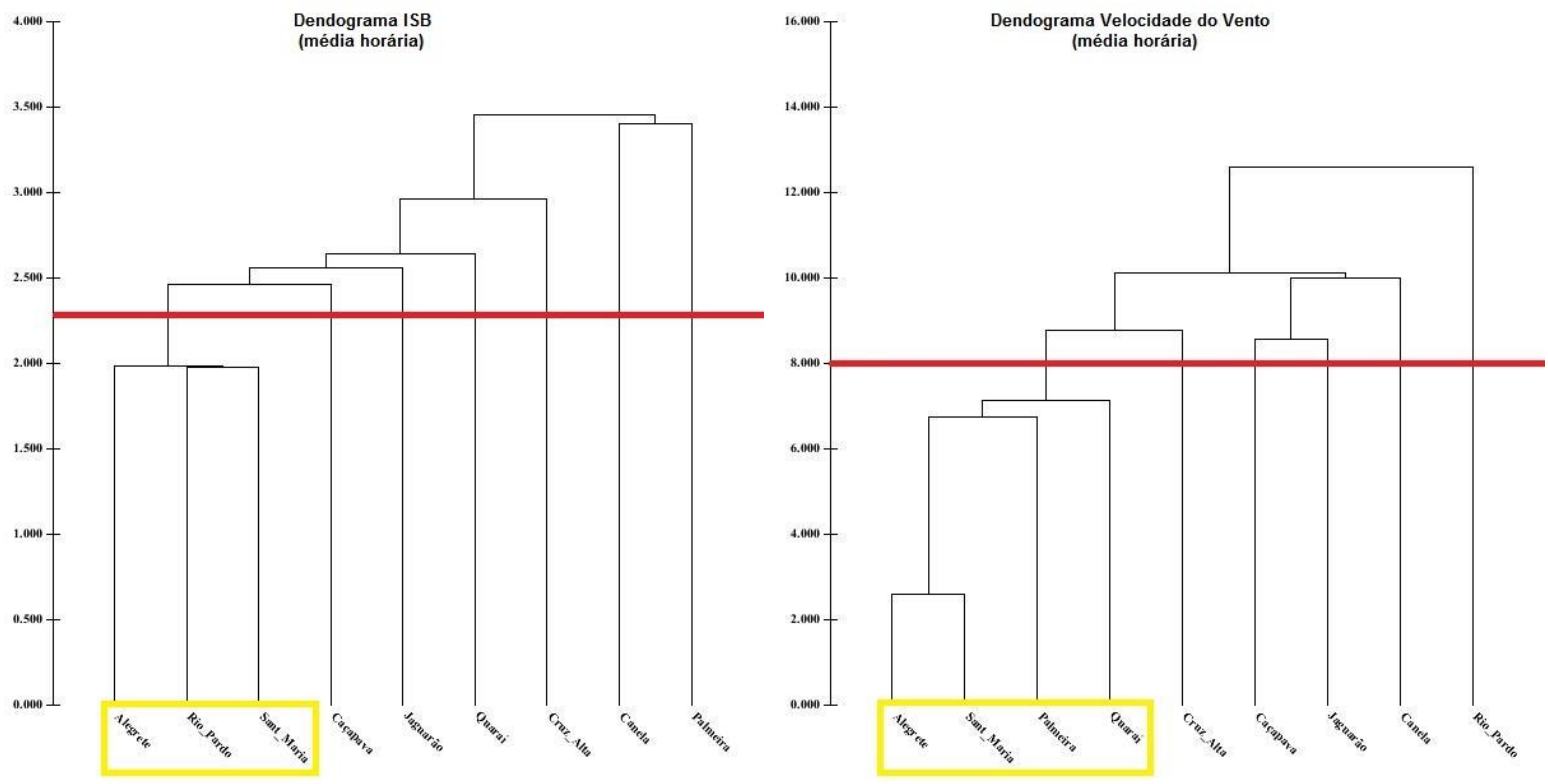

Figura 3 - Dendrogramas de grupos homogêneos de acordo com as médias horárias das variáveis observadas.

Apenas para o agrupamento da variável temperatura do ar houve um número maior de estações meteorológicas agrupadas, sendo um grupo com duas estações e outro com sete estações meteorológicas homogêneas. Também se verificou um grupo com duas estações meteorológicas na análise de agrupamento para a umidade relativa do ar; um grupo contendo três estações meteorológicas para o agrupamento da variável ISB; e um grupo com quatro estações meteorológicas na análise de agrupamento para a velocidade do vento.

A não formação de agrupamentos consistentes nos dendrogramas, associada aos baixos coeficientes de correlação cofenética calculados, em uma primeira análise, demonstram que para a escala temporal horária não há transferência de informações/energia de escalas superiores para escalas inferiores do clima, como foi observado nos trabalhos de Jajcay et al. (2016), Chekroun et al. (2011) e Groth e Ghil (2011; 2015).

Como não foi comprovada influência das escalas superiores sobre as escalas inferiores do clima a partir da análise das médias horárias para as variáveis observadas, optou-se pela análise dessas mesmas variáveis quanto às suas médias diárias. Para tanto, calculou-se novamente o coeficiente de correlação cofenética para todos os métodos de agrupamento disponíveis e verificou-se, novamente o método de encadeamento único como o que fornece os melhores valores de correlação cofenética, apontando valores superiores a 0,7 para todas as variáveis observadas (Tabela 3 ). 
Tabela 3 - Coeficientes de correlação cofenética dos agrupamentos por método de encadeamento único para a média diária das variáveis observadas.

\begin{tabular}{cc}
\hline Agrupamentos (análise diária) & $\begin{array}{c}\text { Coeficiente de Correlação } \\
\text { Cofenético }\end{array}$ \\
\hline ISB & 0,773 \\
Tar & 0,825 \\
UR & 0,953 \\
V & 0,891 \\
\hline
\end{tabular}

Elaborada pelo autor.

A análise dos agrupamentos gerados a partir dos dados médios horários para a Tar, UR, V e ISB demonstrou grande influência do clima sub-regional e regional, bem como das condicionantes geomorfológicas, tais como as compartimentações morfoestruturais do estado. Assim, para uma melhor interpretação das análises dos agrupamentos, optou-se pelas espacialização das estações meteorológicas agrupadas e análise integrada de suas respectivas localizações geográficas com as unidades geomorfológicas do Rio Grande do Sul (MÜLLER FILHO, 1970) e as diferentes tipologias climáticas do estado (ROSSATO, 2014).

Rossato (2014) classifica o Rio Grande do Sul quanto à tipologia climática em área de domínio do clima subtropical, subdividido em quatro tipos principais: Subtropical I - pouco úmido (subtropical Ia - pouco úmido com inverno frio e verão fresco, e subtropical Ib - pouco úmido com inverno frio e verão quente); Subtropical II - mediamente úmido com variação longitudinal das temperaturas médias; Subtropical III - úmido com variação longitudinal das temperaturas médias; e Subtropical IV - muito úmido (subtropical IVa - muito úmido com inverno fresco e verão quente, e subtropical IVb - muito úmido com inverno frio e verão fresco).

Já Müller Filho (1970) divide o Rio Grande do Sul em quatro províncias/compartimentos geológicos: o escudo, o planalto, a depressão periférica e a planície costeira. E em relação à compartimentação geomorfológica do estado, que é fortemente condicionada pela geologia regional, o Rio Grande do Sul pode ser dividido em cinco regiões: Planalto da Bacia do Paraná, Planície Litorânea, Escudo Sul-Rio-grandense, Depressão Periférica Sul-Rio-grandense e Cuestas de Haedo (MÜLLER FILHO, 1970).

A Figura 3 apresenta os dendrogramas das análises de agrupamento por meio do método de encadeamento único para as quatro variáveis observadas, bem como a espacialização desses agrupamentos. É possível observar no dendrograma para a média diária do ISB que houve a formação de um grupo contendo seis estações meteorológicas - Santa Maria, Alegrete, Quaraí, Rio Pardo, Caçapava do Sul e Jaguarão -, todas localizadas na metade sul do estado, onde a participação dos sistemas polares é mais frequente em comparação com a metade norte, onde os sistemas intertropicais têm representação um pouco maior (ROSSATO, 2014; SARTORI, 2000). Na análise de agrupamento para o ISB, apenas Canela, Cruz Alta e Palmeira das Missões não agruparam, sendo a primeira localizada na serra gaúcha (região nordeste do Planalto da Bacia do Paraná), com altitude média de $800 \mathrm{~m}$, enquanto as duas últimas localizam-se na região central do Planalto da Bacia do Paraná, com cotas altimétricas entre 500 e $650 \mathrm{~m}$. 
Na perspectiva da análise de agrupamento para o ISB, Santa Maria agrupa-se com praticamente todas as estações meteorológicas localizadas na metade sul do estado em uma escala temporal diária de análise.

O dendrograma da análise de agrupamento para a média diária da temperatura do ar (Figura 4), por sua vez, apresenta dois grupos distintos, um com seis estações meteorológicas - Santa Maria, Palmeira das Missões, Caçapava do Sul, Jaguarão, Rio Pardo e Cruz Alta -, enquanto o segundo grupo é formado apenas por Alegrete e Quaraí.

Das nove estações meteorológicas observadas, apenas Canela não se agrupou em relação à temperatura média diária. Quando observado o mapa da espacialização da análise de agrupamento representada no dendrograma para a temperatura do ar, verifica-se uma clara compartimentação dos grupos, estando as estações meteorológicas de Quaraí e Alegrete, no extremo oeste do estado, assim como Canela, no extremo nordeste, destacadas das demais estações agrupadas. 

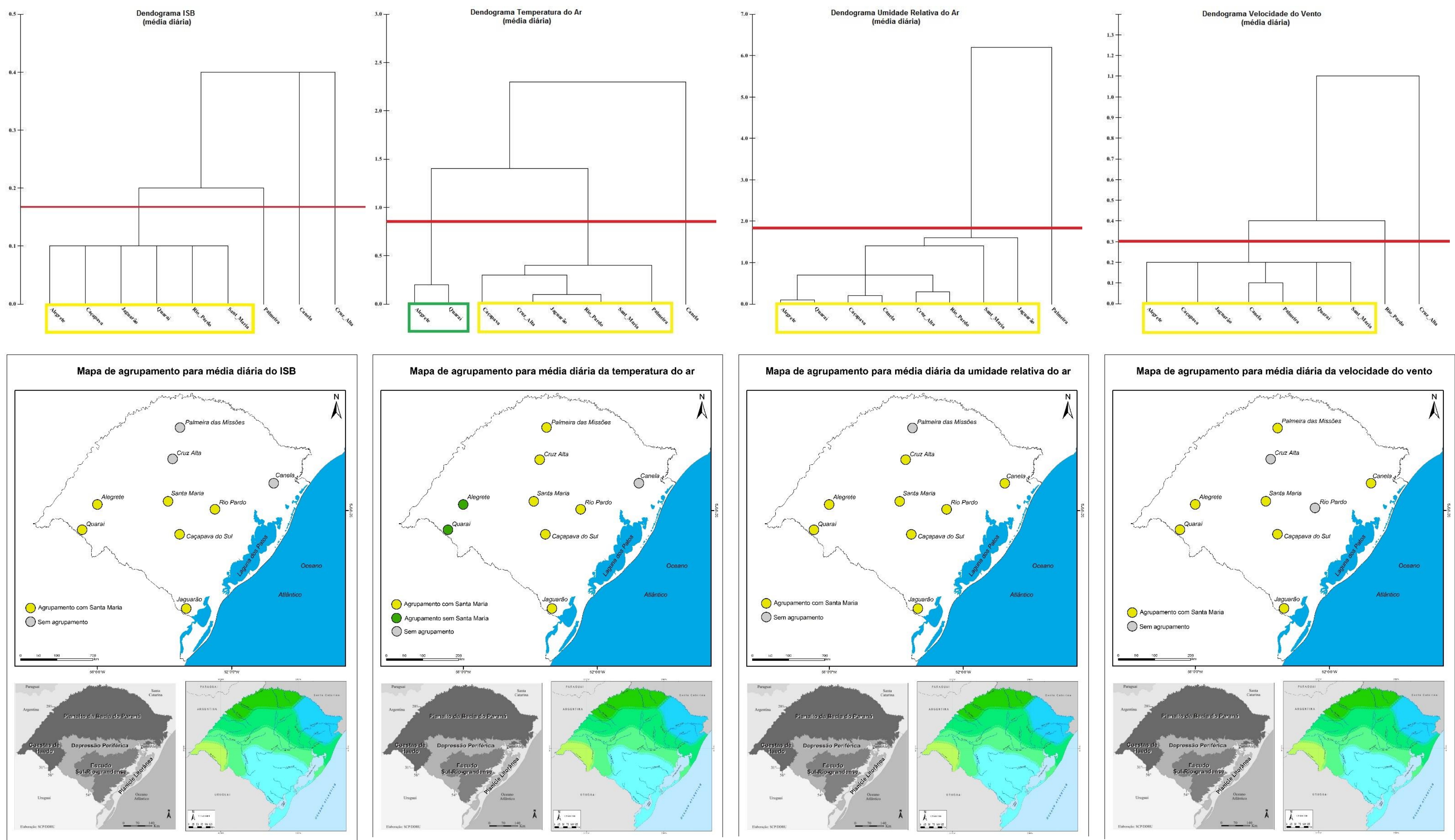

Figura 4 - Dendrogramas e mapas de localização dos grupos homogêneos de acordo com as médias diárias das variáveis observadas. 
Por outro lado, para a umidade relativa do ar, a análise de agrupamento das médias diárias determinou um grande grupo com oito estações meteorológicas: Santa Maria, Caçapava do Sul, Jaguarão, Rio Pardo, Cruz Alta, Quaraí, Canela e Alegrete. Apenas Palmeira das Misssões não foi agrupada. Mais uma vez, ao analisar espacialmente as estações agrupadas, observa-se que, do ponto de vista da tipologia climática do estado (ROSSATO, 2014), Palmeira das Missões, a única estação anômala observada, encontra-se no tipo climático Subtropical IV, muito úmido, com inverno fresco e verão quente, corroborando a disparidade nos dados de UR, o que levou essa estação a não formar agrupamento com as demais.

Em compensação, da perspectiva da UR, Santa Maria agrupa-se com praticamente todas as estações meteorológicas analisadas para o estado em uma escala temporal diária de análise.

Por fim, a análise de agrupamento para as médias diárias de velocidade do vento apresentou, assim como para a UR, um grande grupo com sete estações meteorológicas: Santa Maria, Palmeira das Missões, Caçapava do Sul, Quaraí, Alegrete, Canela e Jaguarão. Apenas Cruz Alta e Rio Pardo mostraramse anômalas e não formaram agrupamento devido aos seus registros de velocidade do vento elevados. Porém, ao analisar espacialmente o agrupamento para essa variável, não há uma explicação regional e/ou subregional para essa definição, uma vez que os aspectos que determinaram as estações de Cruz alta e Rio Pardo como anômalas devem ser atribuídos exclusivamente a feições locais das referidas estações.

Embora tenham sido constatados agrupamentos bem definidos para as médias diárias das quatro variáveis, ainda assim a variável velocidade do vento e a variável temperatura do ar não apresentaram agrupamentos coerentes com os aspectos estruturais do relevo, bem como com a tipologia climática do Rio Grande do Sul. Aspectos esses que são definidores dos climas sub-regionais e regionais. Assim, optou-se por analisar o potencial de influência das variáveis analisadas em discriminar os grupos formados pelas estações meteorológicas por meio de uma análise de componentes principais (PCA).

Primeiramente foi realizada uma análise de PCA para as variáveis temperatura do ar, umidade relativa do ar e velocidade do vento (Figura 5). A análise de componentes principais é dada por dois gráficos, o x-loadings, onde estão dispostas as variáveis analisadas em quadrantes espaciais, e o scores, onde são representadas as distribuições das amostras nos quadrantes em relação às variáveis (x-loadings).

Logo, por meio dos gráficos de x-loadings e scores da Figura 5, é possível observar que as estações meteorológicas de Santa Maria, Rio Pardo, Caçapava do Sul e Jaguarão distinguem-se das demais estações em função da umidade relativa do ar (uma vez que, no score, se encontram deslocadas no mesmo quadrante da UR do x-loadings). Por outro lado, Alegrete, Cruz Alta e Quaraí distinguem-se das demais em função da temperatura do ar, enquanto Palmeira das Missões tem sua distinção determinada tanto pela temperatura como pela velocidade do vento, e Canela distingue-se exclusivamente pela velocidade do vento. 
Ao observar o valor $\mathrm{x}$-expl (variância explicada) dos gráficos, percebe-se que $92 \%$ da diferença entre os dados é explicada pela separação das variáveis no x-loadings e das amostras no score, ou seja, a maior parte das variações existentes entre as estações meteorológicas analisadas é explicada por essa divisão expressa na análise de componentes principais.

Logo, ao observar o mapa da espacialização da análise de componentes principais, é perceptível o condicionamento geomorfológico e tipo climático dos grupos formados, quando comparados com os mapas de unidades geomorfológicas do RS (MÜLLER FILHO, 1970) e de tipologia climática para o estado (ROSSATO, 2014).

Após a realização de uma análise de componentes principais para as médias diárias da temperatura do ar, umidade relativa do ar e velocidade do vento, incluiu-se como variável na análise o Índice Subtropical Brasileiro. Quando inserido o ISB na análise PCA (Figura 6), há uma normalização da variância explicada (x-expl), uma vez que o seu valor cai de $92 \%$ para $55 \%$, o que significa que a separação (variabilidade) das amostras é explicada $55 \%$ pelas quatro variáveis testadas (Tar, UR, V e ISB). Essa normalização ocasionada pela inclusão do ISB como variável para a análise PCA é condicionada devido ao fato de o índice agrupar as demais variáveis (TA, UR e V) em seu resultado final, aumentando a correlação entre elas e diminuindo a variabilidade dos resultados. Assim, é observada a aproximação de todas as estações meteorológicas ao centro do gráfico scores, o que corresponde a uma menor diferença entre essas amostras (estações meteorológicas) quanto às variáveis analisadas (Tar, UR, V e ISB). 
Revista Brasileira de Climatologia

ISSN: 2237-8642 (Eletrônica)
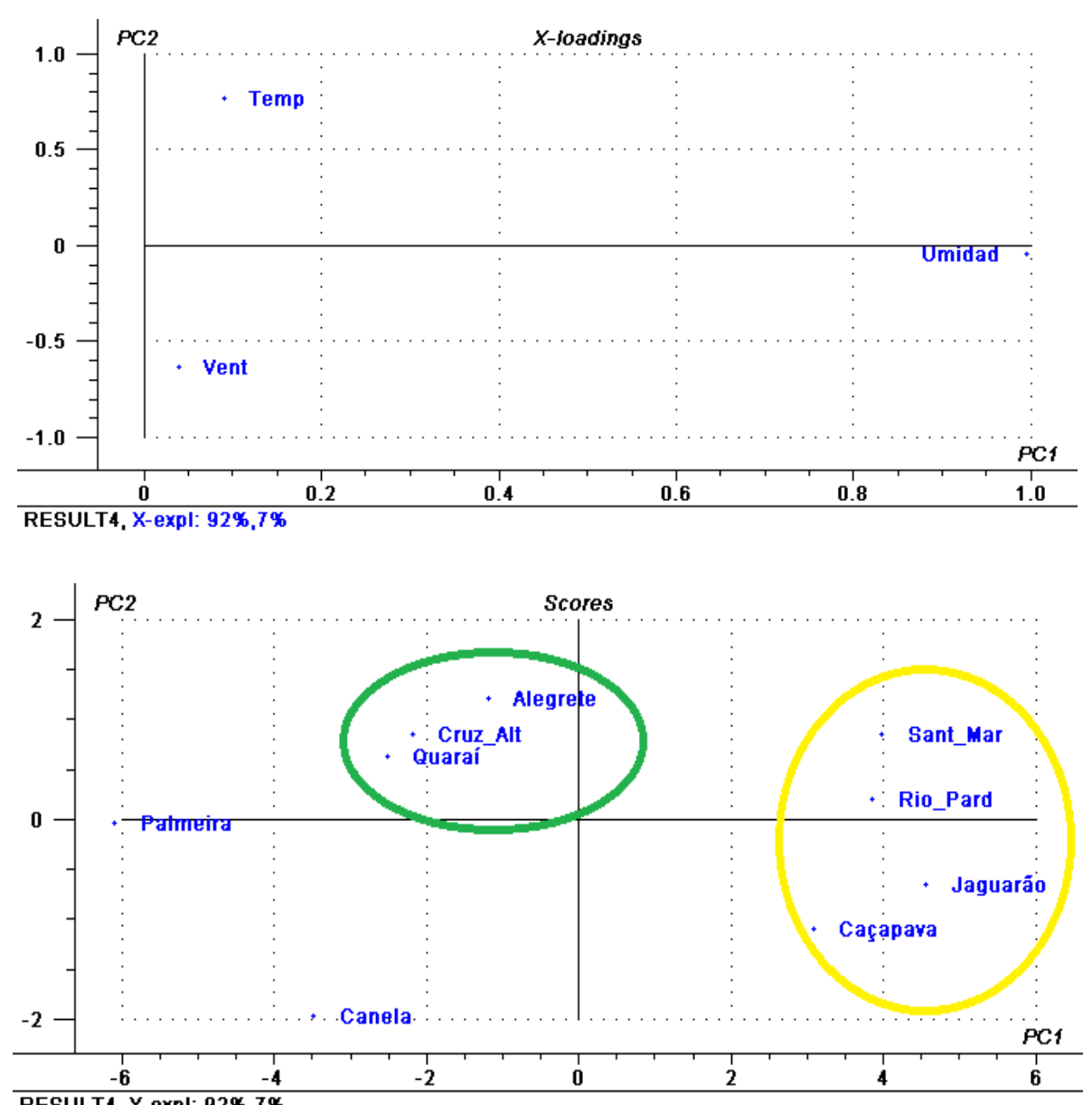

c1
RESULT4, X-expl: 92\%,7\%

Figura 5 - Análise de componentes principais para as variáveis Tar, UR e V das nove amostras observada

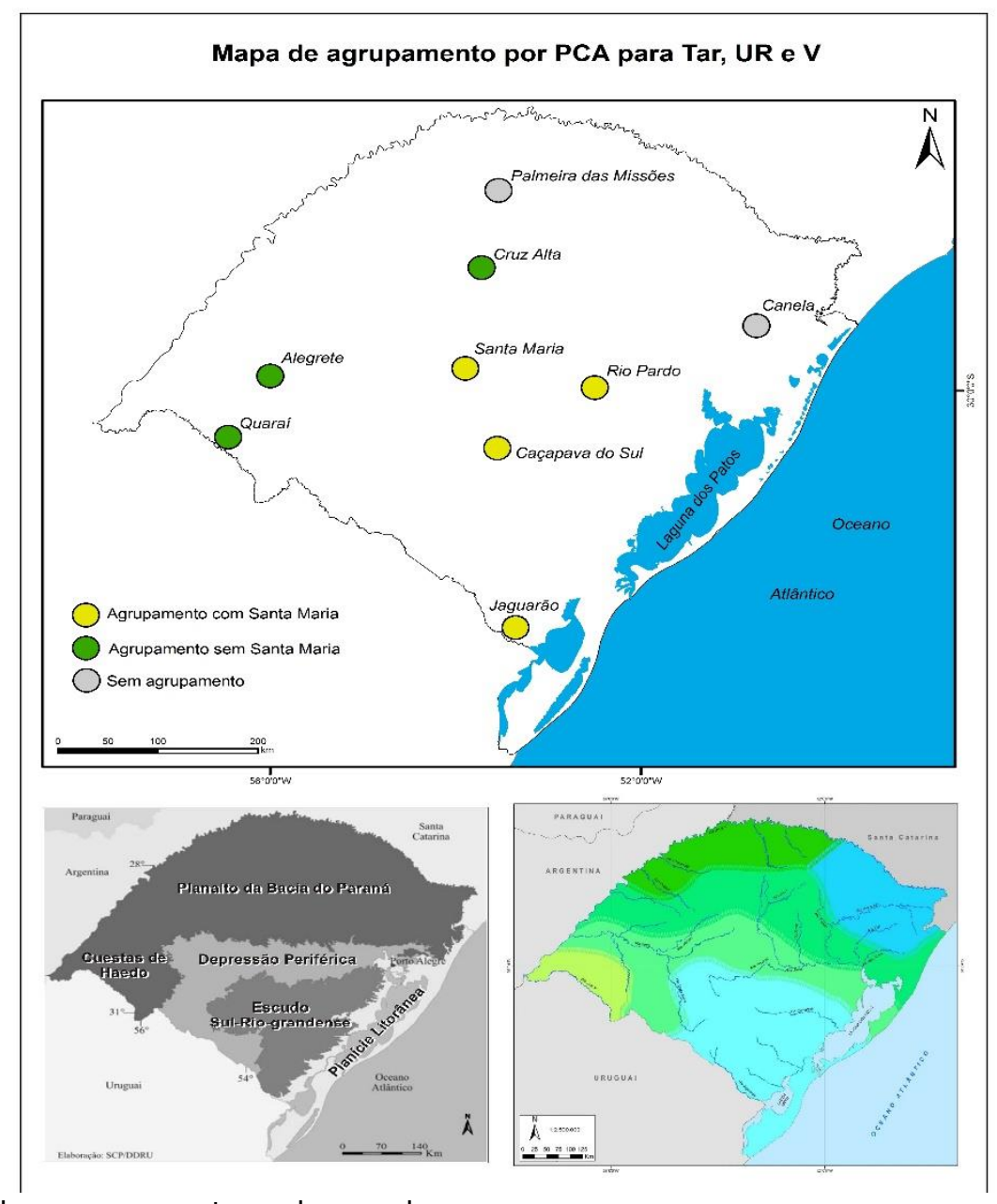


Revista Brasileira de Climatologia

ISSN: 2237-8642 (Eletrônica)
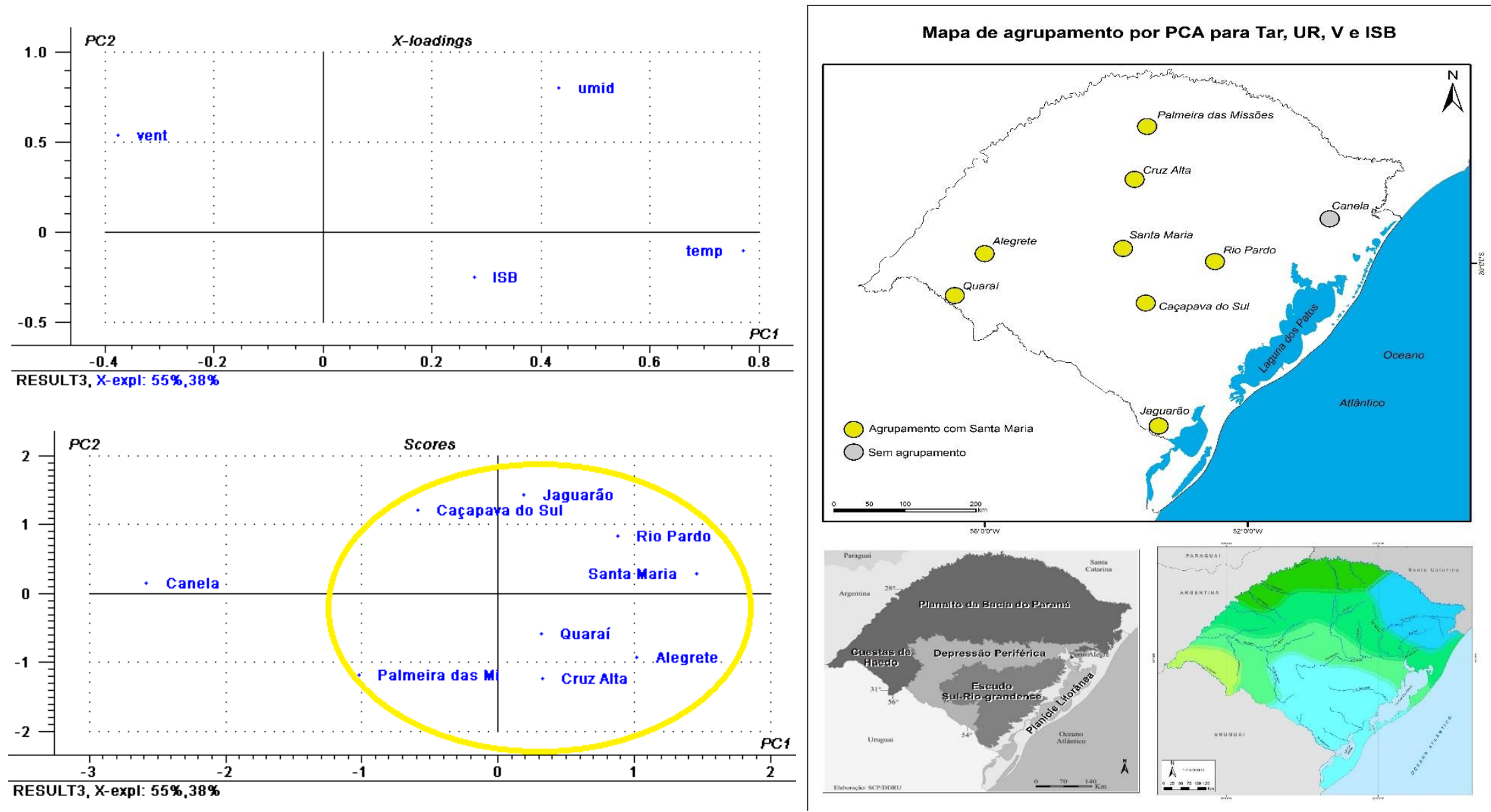

Figura 6 - Análise de componentes principais para as variáveis Tar, UR, V e ISB das nove amostras observadas. 
Apenas a estação meteorológica de Canela não apresentou agrupamento com as demais em função da velocidade do vento, o que se explica, em parte, pela condicionante unidade geomorfológica em que se insere a estação de Canela, na serra gaúcha, com altitude média de $800 \mathrm{~m}$, e também pela tipologia climática dessa região, que, segundo Rossato (2014), encontra-se no tipo climático Subtropical IVb, muito úmido com inverno frio e verão fresco.

\section{CONSIDERAÇÕES FINAIS}

Os resultados encontrados comprovam as análises observadas na literatura que evidenciam a influência da escala temporal de análise na transferência de informações de escalas superiores para escalas inferiores do clima, uma vez que não se obteve similaridade entre as variáveis examinadas para escala temporal horária, enquanto para escala temporal diária observaramse similaridades, as quais foram responsáveis pelos agrupamentos e pela extrapolação escalar da estação de referência (Santa Maria).

Por fim, a análise de componentes principais comprovou de forma eficaz estatística e visualmente a formação de agrupamento e a separação de grupos quando da influência do ISB na análise, o que estabelece a não existência de limites rígidos para as escalas inferiores do clima, sendo estas muitas vezes determinadas pelas escalas superiores em função da análise empregada.

Salienta-se aqui que a análise feita nesta pesquisa foi traçada apenas para os dias de campo trabalhados por Gobo (2018), sendo assim, são necessários novos estudos mais abrangentes e que venham explorar outros tipos de tempo atmosférico, bem como outras escalas de tempo cronológico.

\section{REFERÊNCIAS}

ANDRADE, H. Bioclima humano e Temperatura do ar em Lisboa. Dissertação (Doutoramento em Geografia Física) - Faculdade de Letras da Universidade de Lisboa, 2003.

- Clima Urbano - Natureza, Escalas de Análise e Aplicabilidade. Finisterra, v. 40, n. 80, p. 67-91 2005.

CHEKROUN, M.; KONDRASHOV, D.; GHIL, M. Predicting stochastic systems by noise sampling, and application to the El Nino-Southern Oscillation. Proc. Natl. Acad. Sci. U.S.A., v.108 n.29, p.766-771, 2011.

ELIASSON, I.; SVENSSON, M. K. Spatial temperature variations and urban land use - a statistical approach. In: SVENSSON, M. K. (ed.). Urban climate in relation to land use, planning and comfort. Goteborg University, Department of Earth Sciences, Goteborg, 2002.

GHIL, M.; CHEKROUN, M. D.; STEPAN, G. A collection on 'Climate dynamics: multiple scales and memory effects'. Proc. R. Soc. A, v. 471, issue 2176. 2015.

GOBO, J. P. A.; Bioclimatologia subtropical e modelização do conforto humano: da escala local à regional. Tese (Doutorado) - Faculdade de Filosofia, Letras e Ciências Humanas, USP, São Paulo, 2018. 
GRIMMOND, C. S. B.; OKE, T. R. An evapotranspirationinterception model for urban areas. Water Resour. Res., v. 27, p.1739-1755, 1991.

GROTH, A.; GHIL, M. Multivariate singular spectrum analysis and the road to phase synchronization. PHYSICAL REVIEW E, Vol. 84, Iss. 3 - September 2011.

; GHIL, M. Monte Carlo SSA revisited: Detecting oscillator clusters in multivariate data sets. Journal of Climate, vol. 28, issue 19, p. 7873-7893, 2015.

JAJCAY, N.; HLINKA, J.; KRAVTSOV, S.; TSONIS, A.; PALUS, M. Time scales of the European surface air temperature variability: The role of the 7-8 year cycle. Geophysical Research Letters, v. 43, p.902-909, 2016.

KRÜGER, E. L.; GONZALEZ, D. E. G. Impactos da alteração no albedo das superfícies no microclima e nos níveis de conforto térmico de pedestres em cânions urbanos. Ambiente Construído, v. 16, n.3, p.89-106, jul./set. 2016.

LANDSBERG, H. E. The urban climate. New York: ed. Academic Press, 1981.

MONTEIRO, C. A. F. O estudo Geográfico do Clima. Cadernos Geográficos. Florianópolis, v.1 n .1, maio de 1999.

MOREIRA, P. S. P.; GALVANIN, E. A. S.; DALLACORT, R.; NEVES, R. J. Análise de agrupamento aplicado ao ciclo diário das variáveis meteorológicas nos biomas do estado de mato grosso. Acta Iguazu, v.5 n.1, p.80-94, 2016.

MÜLLER FILHO, I. L. Notas para o Estudo de Geomorfologia do Rio Grande do Sul, Brasil. Publicação Especial 1. Santa Maria: ed. Imprensa Universitária, UFSM, 1970.

OKE, T. R. Boundary layer climates. London: ed. Routledge, 1987.

ORLANSKY, I. Rational subdivision of scales for atmospheric processes. Bull. Amer. Met. Soc., v. 56, p. 527-530, 1975.

PALUŠ, M. Cross-scale interactions and information transfer. Entropy, v. 16, p.5263-5289, 2014.

REGAZZI, A. J. INF 766 - Análise multivariada. Viçosa: Universidade Federal de Viçosa, Centro de Ciências Exatas e Tecnológicas, Departamento de Informática, 166p. 2001.

RIBEIRO, A. G. As escalas do clima. Boletim de Geografia Teorética, v. 23, p.288-294. 1993.

ROSSATO, M. S. Os Climas do Rio Grande do Sul: tendências e tipologia. In: MENDONÇA, Francisco (Org.). Os Climas do Sul: Em Tempos de Mudanças Climáticas Globais. 1. ed. Jundiaí: Paco Editorial, p. 217-271, 2014.

SARAÇLI, S.; DOGAN, N.; DOGAN, I. Comparison of hierarchical cluster analysis methods by cophenetic correlation. Journal of Inequalities and Applications, p.203, 2013.

SVENSSON, M. K.; ELIASSON, I. Diurnal air temperature in built-up areas in relation to urban planning. In: SVENSSON, M. K. (ed.). Urban climate in relation to land use, planning and comfort. Goteborg: Goteborg University, Department of Earth Sciences, 2002. 
TERASSI, P. M. B.; GALVANI, E.; Identification of Homogeneous Rainfall Regions in the Eastern Watersheds of the State of Paraná, Brazil. Climate, v. 5, p.53, 2017.

VALENTIN, J. L. Ecologia numérica: uma introdução à análise multivariada de dados ecológicos. Rio de Janeiro: ed. Interciência, 2000.

VEJMELKA, M.; POKORNÁ, L.; HLINKA, J.; HARTMAN, D.; JAJCAY, N.; PALUŠ, M. Non-random correlation structures and dimensionality reduction of multivariate climate data. Clim. Dynam., v. 44 p. 9-10, 2014.

VICINI, L.; SOUZA, A. M.; Análise Multivariada da Teoria a Prática. Ed. Caderno Didático. Santa Maria: UFSM, 2005.

WANNER, H.; FILLIGER, P. Orographic Influence on Urban Climate. Weather and Climate, v. 9, p.22-28, 1989. 